\title{
The applications of pharmacogenetics
}

\section{to prescribing: what is currently}

\section{practicable?}

Munir Pirmohamed, NHS Chair of Pharmacogenetics, MRC Centre for Drug Safety Science, Department of Pharmacology and Therapeutics, The University of Liverpool

Both during the human genome project ${ }^{1}$ and since its completion interest in pharmacogenetics has increased significantly, evidenced by an increasing number of publications and interest from industry and healthcare systems. Drugs are increasingly prescribed on the basis of genetic (or phenotypic) tests. This article highlights areas in which pharmacogenetics currently plays a role in prescribing (or likely to do so in the near future). Definitions of pharmacogenetics and other relevant terms are given in Table 1.

\section{Pharmacogenetic tests}

Pharmacogenetic tests can be based on phenotype or genotype. Although genotypic tests are usually easier and cheaper, phenotypic tests are more widely used because not all the genotypes that contribute to a phenotype have been defined. Genotypic tests may detect single nucleotide polymorphisms (substitution of one base by another) or more structural variants such as insertions/deletions or copy number polymorphisms. Those variations that determine the level of expression of an mRNA species are also being used, especially in cancers.

Genotypic or phenotypic tests can be used to guide drug choice and/or dosage regimen. A test may predict how efficacious or safe the drug is likely to be. This is relatively straightforward when there is an alternative drug that may be more effective or safe in an individual. Individualising drug dosages, however, is much more difficult.

\section{Determining drug choice}

\section{Cancer therapy}

Personalised therapy is more advanced in cancer than in any other therapeutic area (Table 2). The best example is trastuzumab, which improves diseasefree and overall survival in breast cancer in patients with HER2 gene amplification or overexpression on breast cancer cells. ${ }^{2}$ This adverse prognostic factor, a somatic change, occurs in $20 \%$ of

Table 1. Some relevant definitions.

\begin{tabular}{ll}
\hline Pharmacogenomics* & $\begin{array}{l}\text { The study of variations of DNA and RNA characteristics as } \\
\text { related to drug response } \\
\text { Pharmacogenetics* }\end{array}$ \\
Genomic biomarker* & $\begin{array}{l}\text { response } \\
\text { A measurable DNA and/or RNA characteristic that is an indicator } \\
\text { of a normal biological or a pathogenic process and/or a } \\
\text { response to therapeutic or other interventions } \\
\text { The application of genomic and molecular data to improve the } \\
\text { delivery of healthcare, facilitate the discovery and clinical testing } \\
\text { of new products, and help determine individual predisposition } \\
\text { to a particular disease or condition }\end{array}$ \\
\hline
\end{tabular}

*Definitions adapted from Note for guidance on definitions for genomic biomarkers, pharmacogenomics, pharmacogenetics, genomic data and sample categories (EMEA/CHMP//CH/437986/2006).

**Definition adapted from the US Genomics and Personalised Medicines Act 2007. breast cancers. In patients with colorectal cancer, the proto-oncogene KRAS acts as a downstream signal transducer of epidermal growth factor receptor (EGFR). ${ }^{3}$ Responses to EGFR inhibitory monoclonal antibodies, panitumumab and cetuximab, are better in patients with the wild-type KRAS gene. ${ }^{4}$ Testing for the KRAS gene is now a routine part of the care of patients with metastatic colorectal cancer and is mentioned both in the Summary of Product Characteristics (SPCs) of panitumumab and cetuximab and in National Institute for Health and Clinical Excellence guidance.

\section{Abacavir}

Abacavir, an HIV-1 reverse transcriptase inhibitor, causes a hypersensitivity reaction (skin rash, fever, gastrointestinal and respiratory effects) in about $5 \%$ of patients and is associated with an HLA allele, HLA-B ${ }^{\star 5701}$. Pre-prescription genotyping prevents abacavir hypersensitivity and is cost-effective. ${ }^{5}$ In Europe, screening for HLA-B ${ }^{\star} 5701$ is now mandatory before prescribing abacavir.

\section{Carbamazepine}

Severe immune-mediated adverse effects can be caused by carbamazepine, including Stevens-Johnson syndrome and toxic epidermal necrolysis. In Han Chinese patients this is associated with HLA- $B^{\star} 1502 .{ }^{6}$ The association is phenotype-specific and does not predispose to carbamazepine-induced hypersensitivity syndrome. The SPC recommends testing for HLA-B ${ }^{\star} 1502$ in Chinese and Thai patients before using carbamazepine. However, the positive predictive value is low because the reaction is rare.

\section{Glucose-6-phosphate dehydrogenase deficiency}

The most common enzyme deficiency worldwide is glucose-6-phosphate dehydrogenase (G6PD) deficiency, with many $(>300)$ allelic variants. ${ }^{7}$ It is associated with acute haemolysis on exposure to oxidising drugs such as primaquine, chlorproguanil-dapsone, sulfonamides 
Table 2. Examples of the use of pharmacogenetics in cancer therapy.

\begin{tabular}{|c|c|c|c|}
\hline Drug & Genomic marker & Disease & Comments \\
\hline Trastuzumab & HER2 receptor expression & Breast cancer & Efficacy marker in $20 \%$ of cases \\
\hline Cetuximab or panitumumab & KRAS mutation & Colorectal cancer & More effective with wild-type KRAS \\
\hline Tamoxifen & CYP2D6 & Breast cancer & $\begin{array}{l}\text { Reduced efficacy in poor CYP2D6 } \\
\text { metabolisers }\end{array}$ \\
\hline Imatinib & BCR-ABL translocation & CML & $\begin{array}{l}\text { Also used in treatment of Philadelphia } \\
\text { chromosome-positive ALL and GI } \\
\text { stromal tumours }\end{array}$ \\
\hline Irinotecan & UGT1A1 polymorphism & Colorectal cancer & $\begin{array}{l}\text { Increased risk of neutropenia and } \\
\text { diarrhoea in patients with UGT } 1 \mathrm{~A} 1 * 28 \\
\text { polymorphism }\end{array}$ \\
\hline Mercaptopurine (and azathioprine) & TPMT & ALL & $\begin{array}{l}\text { Increased risk of severe leukopenia } \\
\text { with homozygote variant and } \\
\text { heterozygous individuals }\end{array}$ \\
\hline Fluorouracil & DHPDH & Colorectal cancer & $\begin{array}{l}\text { Increased toxicity in patients with } \\
\text { variant forms of the enzyme }\end{array}$ \\
\hline
\end{tabular}

$\mathrm{ALL}=$ acute lymphoblastic leukaemia; $\mathrm{CML}=$ chronic myeloid leukaemia; DHPDH = dihydropyrimidine dehydrogenase; Gl $=$ gastrointestinal; TPMT = thiopurine methyltransferase; UGT = uridine diphosphate-glucuronosyl-transferase.

and sulfones, nitrofurantoin, nalidixic acid, quinine, flutamide and methylthioninium chloride. Phenotypic tests for G6PD deficiency are recommended before using drugs such as primaquine, but it is not known how often this is done. Testing for G6PD deficiency is also recommended before using rasburicase, a recombinant urate oxidase enzyme used in preventing hyperuricaemia in patients at high risk of tumor lysis syndrome. $^{8}$

\section{Determining drug dosage regimens}

Dosage regimens are currently determined on the basis of population data. This 'one-dose-fits-all' approach leads to variability in drug response, in terms of both efficacy and toxicity. In some cases, dosage depends, at least partly, on genetic factors.

\section{Mercaptopurine}

Mercaptopurine and its pro-drug azathioprine are metabolised by thiopurine methyl transferase (TPMT) which is trimodally distributed. About $10 \%$ of the population are heterozygotes, while the enzyme is absent in one in $300 .{ }^{9}$ Both genotyping and phenotyping can iden- tify heterozygotes and homozygous variants, but the phenotypic test in erythrocytes is more widely used. In homozygotes, agranulocytosis can be avoided by reducing the dose of mercaptopurine or azathioprine. The usefulness of dosage reduction in heterozygotes is less clear and white cell count monitoring has been alleged to be as effective. It is currently recommended that all patients should be tested for TPMT activity before starting azathioprine. ${ }^{10}$

\section{Irinotecan}

Irinotecan is converted to an active metabolite, SN38. This is glucuronidated by UGT1A1, the activity of which is determined by a two base-pair insertion (TA) in the promoter region. Individuals with seven TA repeats $\left({ }^{\star} 28\right.$ allele) have reduced enzyme activity and higher rates of severe neutropenia and diarrhoea than those with the wild-type allele $\left(\mathrm{UGT} 1 \mathrm{~A} 1{ }^{\star} 1\right) .{ }^{11}$ In the USA there is an FDA-approved test for UGT1A1 genotyping, but it is little used because specific dosing instructions are not included.

\section{Warfarin}

There is a 40 -fold interindividual variation in dosage requirements of warfarin. Together with age and weight, variations

\section{Key Points}

Genetic factors can affect the way individuals respond to drugs in terms of efficacy and toxicity

The contribution to drug response is most likely to be multifactorial and multigenic

Genetic factors can be used to guide drug choice and/or dosage regimen

The best examples of gene-guided therapies currently are the expression of the HER2 receptor as a determinant of the efficacy of trastuzumab, and predisposition to abacavir hypersensitivity by HLA-B* 5701

There is a need to test the clinical validity and clinical utility of any pharmacogenetic markers that are developed

KEY WORDS: adverse drug reactions, drug efficacy, drug prescribing, pharmacogenetics 
in two genes, CYP2C9 (which metabolises S-warfarin) and VKORC1 (pharmacological target: vitamin $\mathrm{K}$ epoxide reductase), account for $50 \%$ of the variance in dosage requirements. ${ }^{12}$ Dosing algorithms incorporating genetic and clinical factors have been developed $^{13}$ but, although testing is mentioned on the label in the USA, as for irinotecan there is no dosage guidance. Routine use is not currently recommended. Randomised controlled trials are underway.

\section{Conclusions}

Genotype-guided prescribing is now routine in a few cases and should increase in coming years. It will probably be most useful for drugs with a narrow therapeutic index in the management of cancers and in drug safety. A House of Lords report has highlighted the need to develop this field. ${ }^{14}$ It will be crucial to test the clinical validity and usefulness of any pharmacogenetic markers that are developed.

\section{Acknowledgments}

The support of the UK Department of Health is acknowledged through the NHS Chair of Pharmacogenetics Research Programme.

\section{References}

1 Meyer UA. Pharmacogenetics - five decades of therapeutic lessons from genetic diversity. Nat Rev Genet 2004;5:669-76.

2 Smith I, Procter M, Gelber RD et al. 2-year follow-up of trastuzumab after adjuvant chemotherapy in HER2-positive breast cancer: a randomised controlled trial. Lancet 2007;369:29-36.

3 Walther A, Johnstone E, Swanton C et al. Genetic prognostic and predictive markers in colorectal cancer. Nat Rev Cancer 2009:9:489-99.

4 Jimeno A, Messersmith WA, Hirsch FR, Franklin WA, Eckhardt SG. KRAS mutations and susceptibility to cetuximab and panitumumab in colorectal cancer. Cancer J 2009;15:110-3.

5 Waters LJ, Mandalia S, Gazzard B, Nelson M. Prospective HLA-B ${ }^{\star} 5701$ screening and abacavir hypersensitivity: a single centre experience. AIDS 2007;21: 2533-4.
6 Chung WH, Hung SI, Hong HS et al. Medical genetics: a marker for Stevens-Johnson syndrome. Nature 2004;428:486.

7 Beutler E. G6PD deficiency. Blood 1994;84:3613-36.

8 Browning LA, Kruse JA. Hemolysis and methemoglobinemia secondary to rasburicase administration. Ann Pharmacother 2005;39:1932-5.

9 McLeod HL, Krynetski EY, Relling MV, Evans WE. Genetic polymorphism of thiopurine methyltransferase and its clinical relevance for childhood acute lymphoblastic leukaemia. Leukaemia 2000;14:567-72.

10 (No authors listed.) TPMT testing before azathioprine therapy? Drug Ther Bull 2009;47:9-12.

11 Liu CY, Chen PM, Chiou TJ et al. UGT1A ${ }^{\star} 28$ polymorphism predicts irinotecan-induced severe toxicities without affecting treatment outcome and survival in patients with metastatic colorectal carcinoma. Cancer 2008; 112:1932-40.

12 Takeuchi F, McGinnis R, Bourgeois S et al. A genome-wide association study confirms VKORC1, CYP2C9, and CYP4F2 as principal genetic determinants of warfarin dose. PLoS Genet 2009;5:e1000433.

13 Garcia D, Regan S, Crowther M, Hughes RA, Hylek EM. Warfarin maintenance dosing patterns in clinical practice: implications for safer anticoagulation in the elderly population. Chest 2005; 127:2049-56

14 House of Lords Science and Technology Committee. 2nd Report of Session 2008-09. HL Paper 107-I. Genomic medicine. London: The Stationery Office Limited, 2009. www.publications. parliament.uk/pa/ld200809/ldselect/ ldsctech/107/107i.pdf (last accessed 31 July 2009).

Address for correspondence:

Professor M Pirmohamed, MRC Centre for Drug Safety Science, Department of Pharmacology and Therapeutics, The University of Liverpool, Ashton Street, Liverpool L69 3GE.

Email: munirp@liverpool.ac.uk

\section{Erratum}

\author{
Fogo A, du Vivier A. The
} cutaneous manifestations of haematological malignancy. Clin Med 2009;4:366-70.

Please note figure 3 on page 368 of the August issue of Clinical Medicine was reproduced incorrectly. This was due to an technical error which occurred during the typesetting process.

The correct version of figure 3 is reproduced below.

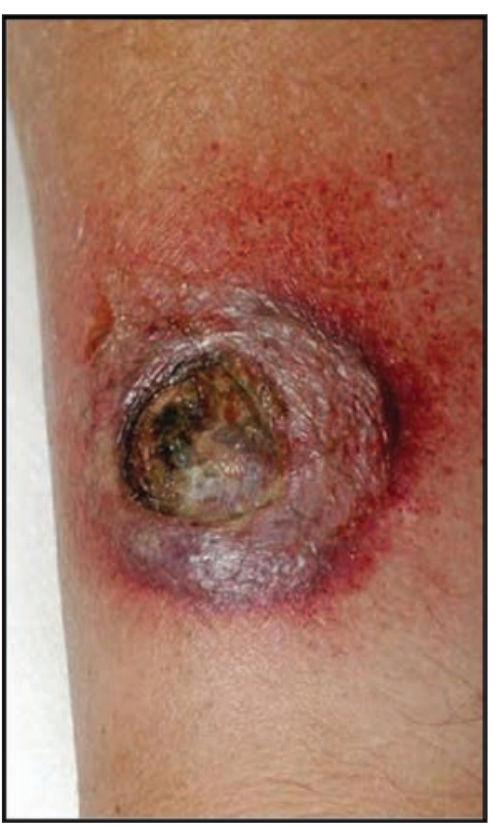

Fig 3. Pyoderma gangrenosum. An 'infection' developed at the site of the Hickman line and at other venous access sites in this patient. It was debrided until a haematologist made the correct diagnosis and a biopsy was performed. The lesions responded dramatically to steroids. 